\title{
Efficacy of the "Pick and Roll" Offense in Top Level European Basketball Teams
}

\author{
by \\ Christos Marmarinos ${ }^{1}$, Nikolaos Apostolidis ${ }^{1}$, Nikolaos Kostopoulos ${ }^{1}$, \\ Alexandros Apostolidis ${ }^{1}$
}

Team offense in basketball games consists of a set of offensive actions carried out with the cooperation of two or more players. Of these actions, the most commonly used in the last decade is the on-ball screen called the "pick and roll." The aim of this study was to analyze all of the pick and rolls conducted in the Euroleague championship from all of the 24 participating teams and to investigate the possible relationships between success in the pick and roll and overall success of the teams. For this purpose, 12,376 pick and rolls from 502 matches were analyzed and classified in categories according to the end result of the offensive possession. The results showed that the most effective type of pick and roll offense was when a shot was attempted after 2 passes from the pick and roll occurrence, followed by the screener's shot when he rolled to the basket. Additionally, linear regression analysis confirmed that pick and roll effectiveness could predict the final classification of the teams. Conclusively, coaches of the high level European clubs should focus on training the players to the most efficient phases of the pick and roll offense, so that the chances of winning the championship to be maximized.

Key words: performance analysis, screen, offensive efficiency, basketball.

\section{Introduction}

Basketball is characterized as an invasion team sport, where two teams dispute on a common field and the main objectives are to score goals, or points, and to prevent the opponent from scoring goals through individual, group and team actions (Grehaigne et al., 1997; Hughes and Bartlett, 2002; Lames, 2006; Lebed, 2007). Among these team actions, the better the collective organization between players, the higher the probability of match success.

Additionally, basketball is a team game requiring various types of interplay, structured or not, for both defense and offense. Sub-phases of interpersonal, attacker-defender, 1-on-1 dynamics exist in basketball (Passos et al., 2011). However, as the game progresses over time, more complex forms are being observed. In the present study, a situation that involves more agents will be analyzed, particularly a 2-on-2 offense-defense interplay in which, in addition to the offensive ball handler, a second player is placing himself in the trajectory of the defender of his ball-handling teammate (screen) (Lamas et al., 2011). In this way the screener is facilitating the ball handler to gain an unobstructed path to the basket. This action, one of the simplest forms of two players working together in a basketball game, is predominantly called the pick and roll (PNR) (Jimenez Sanchez et al., 2009).

As research has shown, decisions and actions of subsystems in large organizations can be modeled as chaotic. Very few outcomes for system behavior can be completely prescribed in advance (Passos et al., 2011). Having that in mind, during the construction of the PNR, teams aim to gain not only from the ball handler's execution,

1 - School of Physical Education \& Sport Science, National and Kapodistrian University of Athens. 
but also from a possible and appropriate pass (Sachanidi et al., 2013) to the directly involved screener (mostly a power forward or center) (Jimenez Sanchez et al., 2009) or to the indirectly involved players who are away from the action, spotted up on the floor.

This form of offensive tactic is used at every level of basketball competition from pickup games to the most recent men's world basketball championships (Wang et al., 2009; Bi Zhong-Chun et al., 2004). Verifying the empirical observations by many coaches, $34.8 \%$ of the dynamic offense used to create spacing among the top 8 national teams in the 2008 Olympics were PNR related (Lamas et al., 2011).

Regardless of the offensive system utilized, the majority of the offensive possessions include some type of the PNR (Hucinski and Tymanski, 2006). It is safe to say, based on previous research, that the PNR is perhaps the most-used tactical pattern during a game in modern basketball (Vaquera et al., 2013). It is possibly the only offensive element that is present as an indicator of performance in all types of matches (Mavridis et al., 2009).

In recent years, there have been numerous efforts to analyze tendencies in basketball. Predominantly, relationships between gamerelated statistics have been sought (Csataljay et al., 2009; Ibanez et al., 2008).

Others have used possessions as an indicator for the explanation of wins and losses (Sampaio and Janeira, 2003). Qualitative attempts to understand variables that are associated with winning have also been applied. Findings suggest that the better a team has built its offense and defense, the more chances exist for overall success (Trninic et al., 2002).

The next step was to investigate which actual offensive situations were connected to success. Some researchers have examined the timing-related factors (fast breaks, secondary fast breaks, organized offenses) (Tsamourtzis et al., 2005), whereas others have examined the spacingrelated factors, e.g., of the inside game (Mavridis et al., 2009). However, the PNR has the highest frequency of occurrence of all offensive types and space-creation dynamics (Lamas et al., 2011). Over the last 10 years, an increase of on-ball screens has been observed and this fact could be a factor connected with an increase in overall efficiency of high-level teams (Strumbelj et al., 2013).

Researchers have viewed the importance of the PNR in a team's offense and started analyzing the PNR (Ibanez et al., 2008; Lamas et al., 2011; Polykratis et al., 2010; Vaquera et al., 2013). Behavioral, contextual and evaluative variables have been examined (Mavridis et al., 2009). There has also been research conducted to inquire into significant differences in the efficiency of the PNR for a single team in a men's world championship (Polykratis et al., 2010). Additionally, poor performance in the PNR was considered to be significant for the failure of another team in the 2012 Olympic Games (Linggang, 2013).

In an effort to provide players and coaches with information about the game, advanced computer engineering has also been utilized. The PNR is used as a data mining factor to analyze basketball tactics (Li-gang and $\mathrm{Fu}$ shun, 2013).

According to the aforementioned literature, there is a lack of studies regarding the analysis of the PNR, especially in high level teams and thus, the purpose of the present study was to provide a comprehensive analysis of the PNR used by top European professional basketball teams. Additionally, the possible relationship between success in the PNR and the overall success of the teams was also investigated.

\section{Material and Methods}

\section{Sample}

The sample consisted of the 24 teams that participated in the 2012-2013 season of the Euroleague Basketball Competition. This league is the highest level of competition in European basketball and second in the world, after the National Basketball Association (NBA).

PNR analysis was performed for all of the games played. The number of games was not the same for all teams. Euroleague operates with a group system; 24 teams start in 4 groups of 6 teams. All of the teams play against each other, home and away, two rounds in each group and a total of 10 games are played for all teams. The last two teams do not qualify for the next round of top 16. In this phase, teams are split into 2 groups of 8 and play a total of 14 games in two rounds. The top 4 teams in each group qualify for the Euroleague playoffs. The best team in a series of 5 
games qualifies to the final round of the competition, the so-called "Final 4". In the final 4 games, two semi-finals are played and the winners play each other for the title, whereas the losers play each other for the third position. In this way, Euroleague teams play from 10 to 31 matches. A total of 502 matches were played and analyzed during that season.

\section{Procedure}

Synergy Sports Systems software (Synergy Sports Technology, San Antonio, Texas, U.S.A., 2013) was used for the analysis. This software company owns the exclusive rights to provide the Euroleague teams with data on all of their games broken down into different categories, regarding the team game or personnel tendencies.

All of the PNR that were used and finished with the offensive team maintaining possession were analyzed. A total of 12,376 PNR plays were selected and examined. The analysis was based on the end result of offensive possession, with PNR plays classified into the following categories: (a) shot by the original ball handler, (b) shot by a non-directly involved player after one pass at the perimeter, (c) shot by a non-directly involved player after two passes at the perimeter, (d) shot by the screener when he is cutting towards the basket (dive in-Poll), and (e) shot by the screener when he is functioning as a perimeter player, moving away from the basket (Pop out).

For each of the 5 categories listed above, the shot was categorized into the following subcategories:

1. successful shot (made) after dribble penetration (lay-up)

2. successful shot after dribble penetration and a free throw (from foul during the shot attempt)

3. unsuccessful (missed) shot after dribble penetration

4. foul during a shot attempt [2 free throws (ft)]

5. turnover

6. successful 2-point mid-range shot [with dribble (pull up) or without (spot up)]

7. successful 2-point mid-range shot and a free throw (from foul during the shot attempt)

8. unsuccessful 2-point mid-range shot (with or without dribble)

9. foul during a shot attempt from mid-range (2 free throws)

10. successful 3-point shot (with or without dribble)
11. successful 3-point shot and a free throw [from foul during a shot attempt (with or without dribble)]

12. unsuccessful 3-point shot (with or without dribble)

13. any of the above, after one or two passes following a PNR

\section{Statistical analysis}

For the statistical analysis, cross-tabulation analysis was used for the categorization of the various types of offense and attempts. Sums, means and percentiles are given. Additionally, Pearson Linear Correlation analysis was conducted for the initial examination of the relationship between PNR effectiveness and the final classification of the teams and also, step by step regression analysis was applied to explore the possible power to predict the final team classification based on PNR effectiveness. For all analyses, SPSS 21.0 was used and the level of significance was set at $p<0.05$.

\section{Results}

\section{Descriptive statistics}

In Table 1, results of the PNR in all 5 selected categories for the 24 teams are presented. A disproportional relationship between PNR use and the points derived from each category was observed.

In the following figure, the results of Table 1 are presented.

In Tables 2 and 3, results of the PNR used for each separate category according to the player who attempted the shot (a ball handler, a receiver of the first pass after the PNR, a receiver of the second pass after the PNR, a roll man, a pop man) and the means of execution as a percentage of total attempts are presented.

In Table 4 , classification of the 24 teams that participated in the Euroleague during the 20122013 season is presented. The table also includes the percentage of PNR successes for each team. The results were published by Euroleague Basketball and are the official results for the season.

\section{Linear correlation}

Linear Pearson correlation analysis was applied to determine the relationships between the variables of the final classification and the percentage of PNR effectiveness. A significant linear correlation $(\mathrm{r}=-0.41, p<0.05)$ was detected. 
This finding indicates that the final classification is correlated with the effectiveness of PNR use. In this case, further exploration of the relationship between the two variables was needed. The negative correlation is shown due to the ascending way in which the team classification is numbered.
Regression analysis

Additionally, linear stepwise regression analysis of the variable "PNR effectiveness" was performed for the variable's ability to predict the final classification of the teams. The results $(\mathrm{F}=4.395, p=.048)$ are significant and the score of $\mathrm{R}^{2}$ $* 100=17$ expresses the common variance of the two variables. In this way, $17 \%$ of the teams' final classification can be explained by their effectiveness in PNR offense.
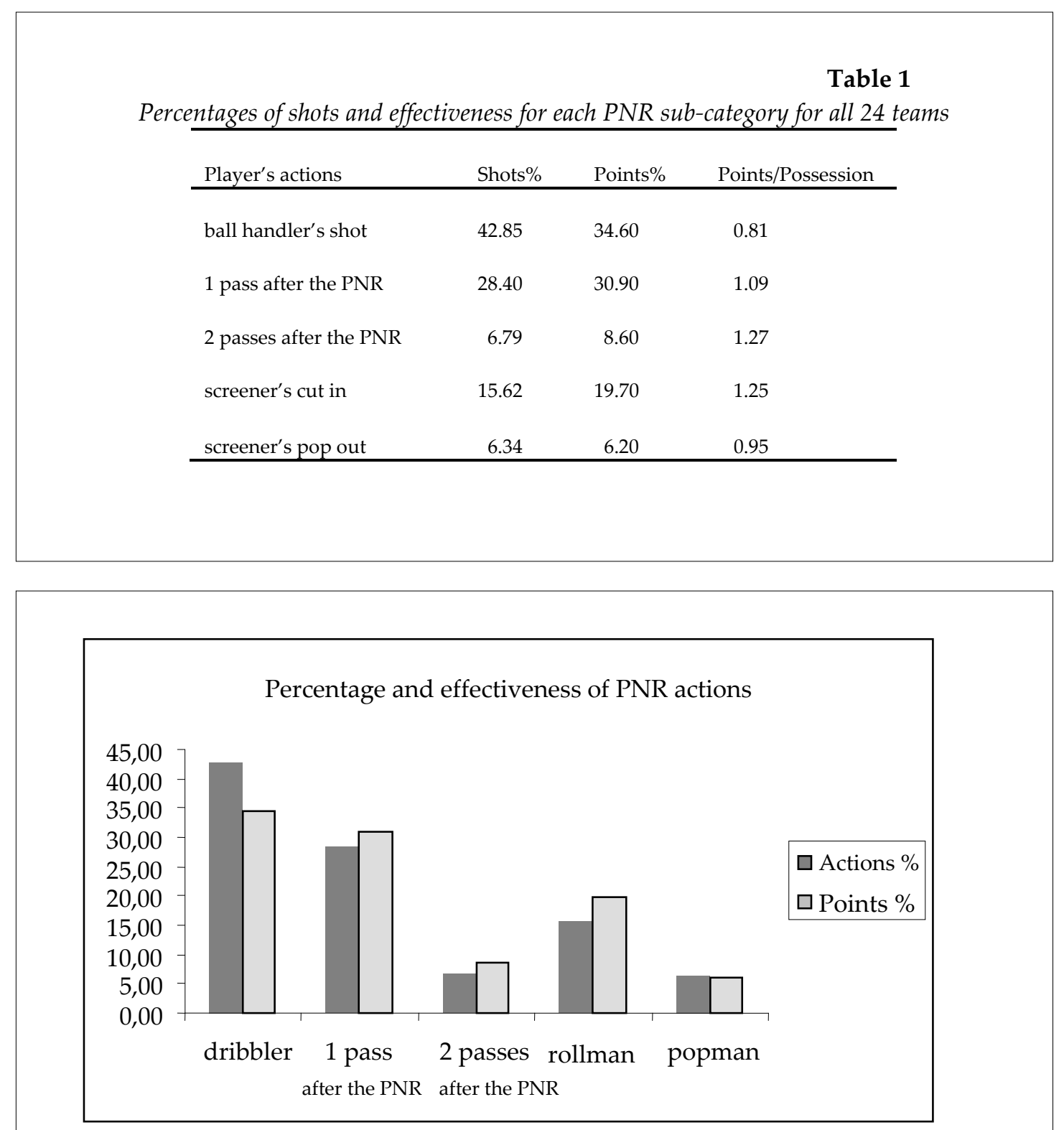

Figure 1

Percentage of PNR use and the effectiveness of PNR actions 
Table 2

Percentage of perimeter player's shots per category for all 24 teams

\begin{tabular}{|c|c|c|c|c|c|c|}
\hline \multirow[b]{2}{*}{ Type of offense } & \multicolumn{2}{|c|}{ Dribbler actions } & \multicolumn{2}{|c|}{ Actions, 1 pass after PNR } & \multicolumn{2}{|c|}{ Actions, 2 passes after PNR } \\
\hline & Shots $\%$ & Points \% & Shots $\%$ & Points $\%$ & Shots $\%$ & Points $\%$ \\
\hline made lay-up & 10.85 & 31.4 & 4.21 & 9.1 & 3.33 & 6.1 \\
\hline made lay-up $+1 \mathrm{ft}$ & 1.15 & 3.7 & 0.23 & 0.6 & 0.12 & 0.3 \\
\hline missed lay-up & 15.02 & & 6.91 & & 4.76 & \\
\hline $2 \mathrm{ft}$ from lay-up & 8.55 & 9.9 & 3.01 & 2.6 & 1.19 & 0.9 \\
\hline made $2 p$ pull up shot & 7.33 & 21.1 & 2.56 & 5.5 & 3.21 & 5.9 \\
\hline made $2 \mathrm{p}$ pull up shot $+1 \mathrm{ft}$ & 0.15 & 1.3 & 0.03 & 0.1 & 0.12 & 0.3 \\
\hline missed $2 \mathrm{p}$ pull up shot & 11.70 & & 4.41 & & 2.73 & \\
\hline $2 \mathrm{ft}$ from pull up shot & 0.58 & 0.5 & 0.37 & 0.3 & 0.00 & \\
\hline made $3 p$ pull up shot & 7.33 & 31.7 & 0.74 & 2.4 & 0.83 & 2.3 \\
\hline made $3 p$ pull up shot $+1 \mathrm{ft}$ & 0.06 & 0.4 & 0.03 & 0.1 & 0.00 & \\
\hline missed $3 p$ pull up shot & 14.32 & & 1.36 & & 1.43 & \\
\hline Turnover & 22.95 & & 8.42 & & 4.28 & \\
\hline made $2 p$ spot up shot & & & 8.59 & 18.6 & 8.68 & 16 \\
\hline made $2 p$ spot up shot $+1 \mathrm{ft}$ & & & 0.65 & 1.9 & 0.48 & 1.2 \\
\hline missed 2 p spot up shot & & & 7.71 & & 7.02 & \\
\hline made $3 p$ spot up shot & & & 17.80 & 57.4 & 23.78 & 65.8 \\
\hline made $3 p$ spot up shot $+1 \mathrm{ft}$ & & & 0.11 & & 0.00 & \\
\hline missed $3 p$ spot up shot & & & 31.36 & & 36.39 & \\
\hline $2 \mathrm{ft}$ from spot up shot & & & 1.51 & 1.4 & 1.66 & 1.2 \\
\hline
\end{tabular}

Table 3

Percentage of screener shots per category for all 24 teams

\begin{tabular}{lcccc}
\hline & Popman actions & Rollman & actions \\
\hline Type of offense & Attempts\% & Points\% & Attempts\% & Points $\%$ \\
\hline made lay-up & 20.00 & 49.3 & 41.65 & 77.8 \\
made lay-up +1ft & 0.76 & 3.5 & 4.60 & 12.1 \\
missed lay-up & 30.06 & & 27.70 & \\
2ft from lay-up & 4.84 & 4.8 & 13.49 & 10.1 \\
Turnover & 9.17 & & 12.56 & \\
made 3p shot & 11.34 & 41.8 & & \\
made 3p shot $+1 \mathrm{ft}$ & 0.13 & 0.6 & & \\
missed 3p shot & 23.69 & & & \\
\hline
\end{tabular}




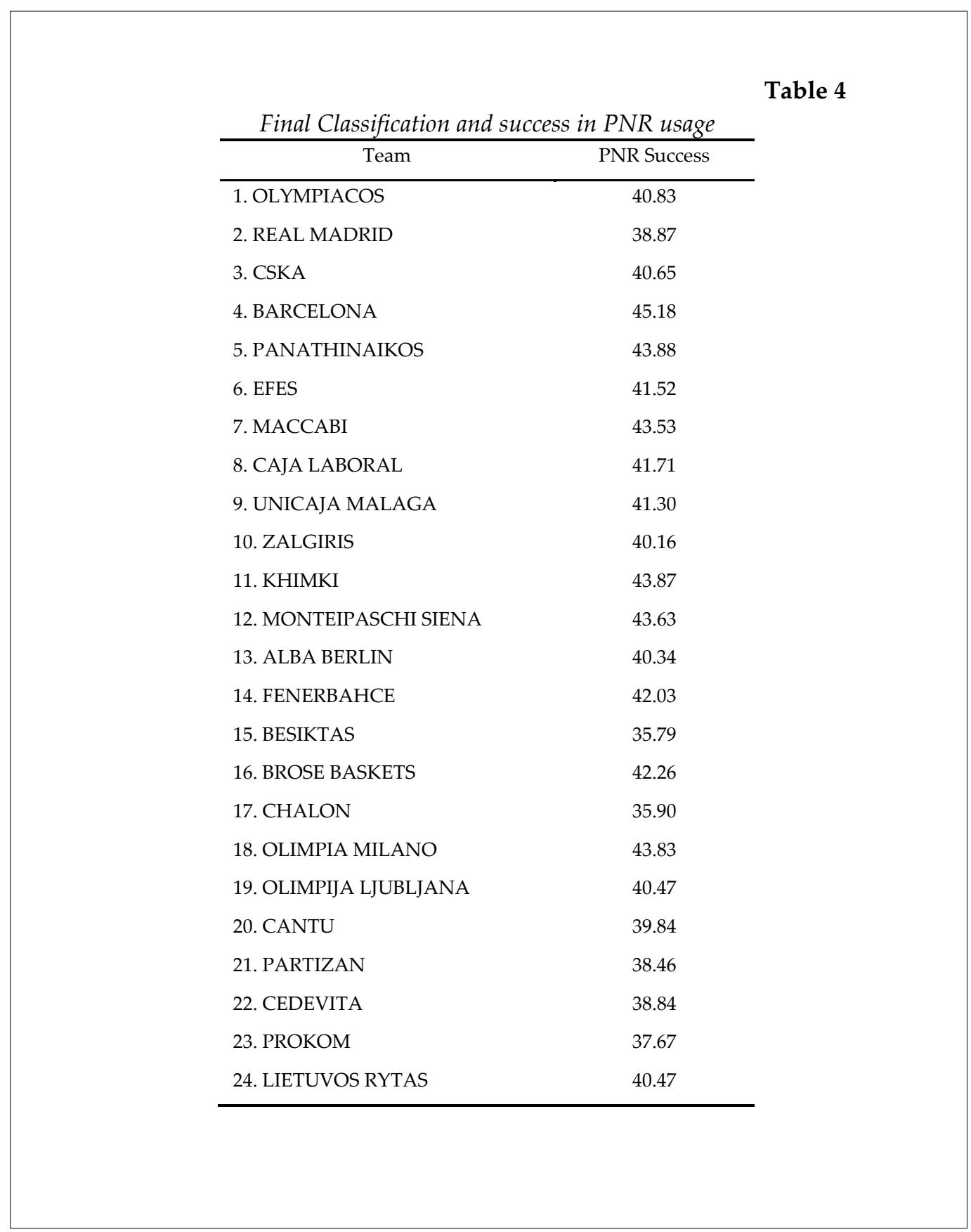

\section{Discussion}

The need to set screens during offensive play has been well-documented from the earliest stages of basketball. The columns of indoor gyms were the first "screens" ever used. The offensive player would skillfully lead his defender right at the column to gain an unobstructed path to the basket.

Recently, many things have changed regarding basketball tactics. Players have become more athletic with much better technical skills.
Coaches have used the PNR as the main offensive pattern at many different levels of competition. The necessity to increase the effectiveness of the PNR is more than obvious. Accordingly, it could be observed that the Euroleague Champions, Olympiacos, have used the PNR as the closing offensive maneuver in $41 \%$ of its total possessions. This finding is in accordance with Lamas et al.'s (2011) research which indicated that approximately $35 \%$ of the dynamic offense used to create spacing among the top 8 national teams in the 2008 Olympics were PNR related. 
Therefore, it is necessary to study which PNR options are more effective. Additionally, we would like to know whether the effectiveness in the use of this offensive tactic can predict the final classification in a basketball league.

Shots by a ball handler following the PNR are the most commonly used PNR tactic, with almost $43 \%$ of shots following the PNR being taken by the ball handler. Players who are spot up and receive the first or second pass take $28 \%$ and $7 \%$ of the shots following the PNR, respectively. At the same time, the screener will take $16 \%$ and $6 \%$ of the shots following the PNR when he rolls in or pops out of the basket, respectively.

Those results appear reasonable. For the most part, coaches ask their players to be aggressive and attack the basket at every opportunity. This tactic often forces the ball handler to take a shot following the PNR. The tactic also explains that a pass to the perimeter $(35 \%)$ results in a shot more often than a pass to the screener (22\%). Defensive players always have a tendency to cover the player closest to the basket first.

However, the 5 PNR execution subcategories are not equally effective. Specifically, the ball handler finishes about $43 \%$ of PNR plays, but this accounts for only $34.60 \%$ of the total points scored from PNR plays. A similar trend is seen when the screener goes away from the basket (pop). Although, the latter does not show a great difference in its occurrence and the points for which it accounts $(6.34 \%$ usage with $6.20 \%$ of the total points scored). On the other hand, the largest percent of points is scored when a shot is taken after one pass to the perimeter (30.90\% of the total points scored and $28.40 \%$ of the PNR plays). After 2 passes, we may observe similar results $(8.60 \%$ of the points scored from $6.79 \%$ of the PNR plays). A pass to the screener when he rolls in the point produces $19.70 \%$ of points scored following the PNR and accounts for $15.62 \%$ of PNR plays. Thus, it is clear that it is more effective for a team to pass to the perimeter (after 1 or 2 passes) or to the screener, when he moves close to the rim.

Additionally, for the means of execution, we observe the following: (a) the ball handler is most effective when he drives the ball to the basket $(10.85 \%)$, when he goes to the line for 2 free throws $(8.55 \%)$, when he takes a mid-range 2- point shot $(7.33 \%)$ or a 3-point shot $(7.33 \%)$. However, such tactics also lead to the most turnovers, allowing $22.95 \%$ of the possessions to be turned over to the opponent; (b) the perimeter player who receives the pass for a shot finishes the play more effectively with a spot up 3- or 2point shot $(17.8 \%$ and $8.59 \%$, respectively). Such tactics also lead to the fewest turnovers; (c) for shots after an extra pass (2 passes), a spot up 3point shot is the most common tactic $(23.78 \%)$ with the spot up 2-point shot the next most common $(8.68 \%)$; (d) the highest percentage of success occurs when a pass is made to the screener who rolls in. In such cases, the player converts the 2-point shot during a drive at a $41.65 \%$ rate, is awarded free throws $4.65 \%$ of the time and draws a simple shooting foul $13.49 \%$ of the time. Contrarily, he misses the shot $27.70 \%$ of the time and turns the ball over during $12.56 \%$ of the possessions; (e) in the last subcategory, a screener who pops out will convert a 2-point shot $20 \%$ of the time and a 3 -point shot $11.34 \%$ of the time.

In total, the most effective form of PNR attack is a pass to the screener when he rolls in $(59.74 \%)$ followed by a shot after 2 passes $(44.35 \%)$, a shot from the spot up player after 1 pass $(42.68 \%)$, a shot from a pop out situation (37.07\%) and when the ball handler shoots (36\%).

The analysis of correlation among the variables of effectiveness in PNR use and final classification of the teams produced a positive relationship $(\mathrm{r}=-0.41, \mathrm{p}<0.05)$. The two variables correlate, and there is room for further analysis. This analysis considered whether PNR effectiveness can predict the final outcome for a team playing in a league.

Indeed, linear stepwise regression analysis showed $(\mathrm{F}=4.40, \mathrm{p}<0.05)$ that the final classification of a team could be marginally predicted from the success the team had in executing the PNR and this finding is in accordance with Trninic et al.'s (2002) research which observed that the better a team had built its offense and defense the more chances existed for overall success. Particularly, the explanation provided by the square of the correlation coefficient $(\mathrm{R} 2 \approx 0.17)$ is that $17 \%$ of the variance of team's final classification can be explained by the team's efficiency in the PNR. This percentage exceeds the level of significance $(10 \%)$ set by 
Pedhazur (1982).

The purpose of the present investigation was to examine the effectiveness of the PNR when used to end a possession. All such offensive plays used by the 24 teams that participated in the 20122013 season were analyzed. The results of this study can be generalized for such tournaments and high-level teams as all analogous possessions were used instead of sub-samples. The analysis of 502 games and 12,376 PNR plays can provide a relatively safe generalization of the results.
Conclusively, possessions that end with the screener's rolling in the shot and those that end with 2 passes following the PNR are the most effective uses of the PNR. The least successful use of the PNR is when the ball handler shoots. Also, the effectiveness of PNR offense explains a relatively small $(17 \%)$, but significant part of the variance of the final classification of a team.

\section{Acknowledgements}

The authors appreciate the scouting stuff of Olympiacos BC for their hospitality and assistance in videoanalysis of the matches. Special thanks are also addressed to the post graduate students of the Athens Faculty of Physical Education and Sport Science, for their assistance in the categorization of the analyzed phases.

\section{References}

Csataljay G, James N, Hughes M, Dancs H. Performance indicators that distinguish winning and losing teams in basketball. International Journal of Performance Analysis in Sport, 2009; $\quad$ 9: 60-66

Grehaigne JF, Bouthier D, David B. Dynamic-system analysis of opponent relationships in collective actions in soccer. J Sport Sci, 1997; (15)2: 137-149

Hucinski T, Tymanski R. The structure of technical-tactical actions and effectiveness of the youth Polish National Team, European Basketball Vice-Champions. Research Yearbook, 12, 267-271; 2006

Hughes M, Bartlett R. The use of performance indicators in performance analysis. J Sport Sci, 2002; (20)10: 739-754

Ibanez SJ, Sampaio J, Saenz-Lopez P, Gomez MA, Ortega E. Basketball game-related statistics that discriminate between teams' season-long success. European Journal of Sports Science, 2008; 8(6): 369-372

Jimenez Sanchez AC, Calvo AR, Saenz-Lopez Bunuel P, Ibanez Godoy SJ. Decision-making of the Spanish female basketball team players while they are competing. Rev Psicologia Dep, 2009; 18: 369-373

Lamas L, De Rose J, Santana F, Rostaiser E, Negretti L, Ugrinowitch C. Space creation dynamics in basketball offence: validation and evaluation of elite teams. International Journal of Performance Analysis in Sports; 2011; 11: 71-84

Lames M. Modelling the Interaction in Game Sports - Relative Phase and Moving Correlations. Journal of Sports Science \& Medicine, 2006; 5(4): 556-560

Lebed F. A dolphin only looks like a fish: Players' behaviour analysis is not enough for game understanding in the light of the systems approach - a response to the reply by McGarry and Franks. European Journal of Sport Science, 2007; (7)1: 55-62

Li-gang T, Fu-shun W. Design and application of Basketball Tactics Analysis Based on the Database and Data Mining Technology. Paris France: Atlantis Press, 1457-60; 2013

Mavridis G, Tsamourtzis E, Karypidis N, Laios A. The inside game in World Basketball. Comparison between European and NBA teams. International Journal of Performance Analysis in Sport, 2009; (9)2: 157164

Passos P, Milho J, Fonseca S, Borges J, Araujo D, Davids K. Interpersonal distance regulates functional grouping tendencies of agents in team sports. J Motor Behav, 2011; 43: 155-163

Pedhazur EJ. Multiple regression in behavioral research: Explanation and prediction. New York: Holt, Rinehart \& Winston; 1982

Polykratis M, Tsamoutzis E, Mavridis G, Zaggelidis G. Relation of effectiveness in Pick and Roll application 
between the national Greek team and of its opponents during the men's world basketball championship of 2006. Journal of Physical Education and Sport, 2010; 29: 57-67

Trninic S, Dizdar D, Luksic E. Differences Between Winning and Defeated Top Quality Basketball Teams in Final Tournaments of European Club Championship. Collegium Anthropol, 2002; (26)2: 521-531

Tsamourtzis E, Karypidis N, Athanasiou N. Analysis of Fast Breaks in Basketball. International Journal of Performance Analysis in Sport, 2005; (5)2: 17-22

Sachanidi M, Apostolidis N, Chatzicharistos D, Bolatoglou T. Passing efficacy of young basketball players: test or observation? International Journal of Performance Analysis in Sport, 2013; 13: 403-412

Sampaio J, Janeira M. Statistical analyses of basketball team performance: understanding team's wins and losses according to a different index of ball possessions. International Journal of Performance Analysis in Sport, 2003; 3: 40-49

Strumbelj E, Vracar P, Robnik-Sikonja M, Dezman B, Erculj F. A Decade of Euroleague Basketball: an Analysis of Trends and Recent Rule Change Effects. Journal of Human Kinetics, 2013; 38: 183-189

Vaquera A, Cibillo R, Garcia-Tormo J, Morante J. Validation of a tactical analysis methodology for the study of pick and roll in Basketball. Revista de Psicologia del Deporte, 2013; 22(1): 277-281

Wang J, Liu W, Moffit J. Skills and offensive tactics used in pick up basketball games. Percept Motor Skill, 2009; 109: 473-477

Zhong-chun B, Lu-ming G, Qing-hui Y, Shu-guang S. The new trends of Tactics and Technique of the World Basketball - Take the $16^{\text {th }}$ Men's World Basketball Championship as the Example. Journal of Beijing Sport University, 2010

\section{Corresponding author:}

Nikolaos Apostolidis, PhD, Associate Professor

Department of Games and Sports, School of Physical Education \& Sport Science, National \& Kapodistrian University of Athens, Greece

41 Ethnikis Antistasis str. Dafne, 172 37, Athens, Greece

Tel+Fax.: +30210 7276085

E-mail: napost@phed.uoa.gr 